This item was submitted to Loughborough's Research Repository by the author.

Items in Figshare are protected by copyright, with all rights reserved, unless otherwise indicated.

\title{
Thermal and electrical modelling of polymer cored BGA interconnects
}

PLEASE CITE THE PUBLISHED VERSION

PUBLISHER

(C) IEEE

VERSION

VoR (Version of Record)

LICENCE

CC BY-NC-ND 4.0

\section{REPOSITORY RECORD}

Whalley, David C., H. Kristiansen, and F. Guillen Marin. 2019. "Thermal and Electrical Modelling of Polymer Cored BGA Interconnects". figshare. https://hdl.handle.net/2134/4217. 
This item was submitted to Loughborough's Institutional Repository (https://dspace.lboro.ac.uk/) by the author and is made available under the following Creative Commons Licence conditions.

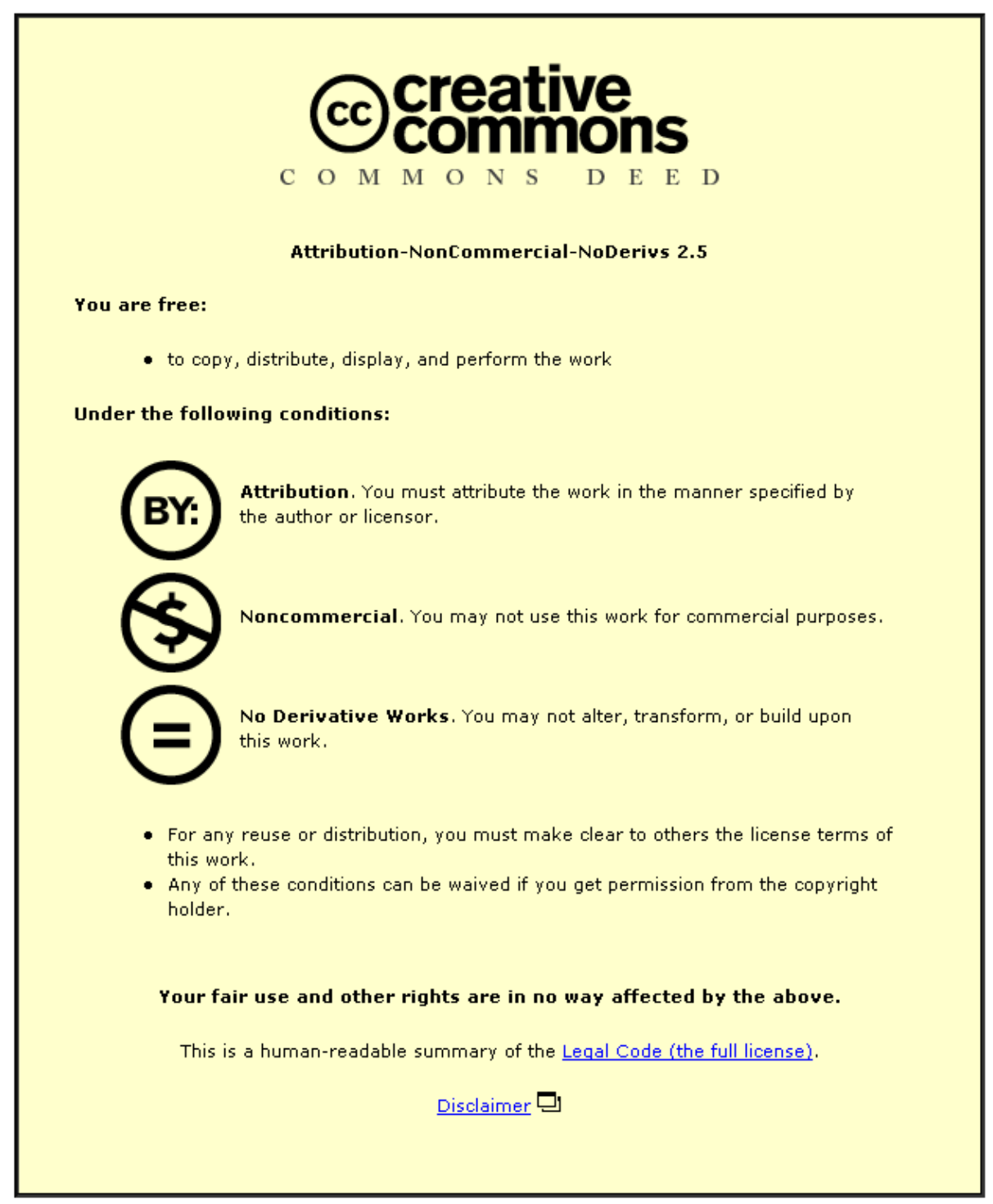

For the full text of this licence, please go to: http://creativecommons.org/licenses/by-nc-nd/2.5/ 


\title{
Thermal and Electrical Modelling of Polymer Cored BGA Interconnects
}

\author{
D. C. Whalley ${ }^{1,2}$, H. Kristiansen ${ }^{1}$ and F. Guillen Marin ${ }^{2}$ \\ ${ }^{1}$ Conpart AS \\ Skjetten, Norway \\ ${ }^{2}$ Wolfson School of Mechanical and Manufacturing Engineering \\ Loughborough University \\ Loughborough, UK
}

\begin{abstract}
Polymer cored BGA/CSP balls have been proposed as a more reliable alternative to solid solder balls for demanding application environments. Their potential advantages are dependant on their increased compliance compared with a solid solder ball, thereby reducing the level of stress imposed on the solder joints during exposure to cyclic thermal loads and impacts. The latter is of particular importance for hand held products assembled using lead free solders, which are much more brittle than traditional tin-lead alloys, but this may also be important for harsh environment applications where tinlead solders are still being used, such as in aerospace and defence electronics applications. The increased compliance of a polymer cored ball may reduce the requirement for underfilling of components in hand held products, and allow adoption of BGA/CSP for safety critical applications in harsh environments. Such polymer cored interconnects are however likely to provide a reduced thermal and electrical conductivity and it is important to ensure any such effects do not impact upon the thermal and electrical performance of the product.

This paper utilises analytical and computational modelling techniques to achieve an understanding of the effect of conductor particle geometry and properties on thermal and electrical performance. Such models offer a route to appropriate materials selection for the polymer spheres and their conductive coatings, and for establishing optimum design parameters such as ball diameter, conductive coating thickness, solder pad diameter, and solder volumes.

The results confirm that the introduction of polymer cored BGA balls will result in some increases in thermal and electrical resistance, but that these changes will have minor impacts on the overall performance of products.
\end{abstract}

\section{Introduction}

Polymer cored balls for use as interconnects in ball grid array (BGA) and chip scale packaging (CSP) have been proposed as a more reliable alternative to the more conventionally used solid solder balls [1]. Their potential advantages are dependant on their increased flexibility compared with a solid solder ball, thereby reducing the level of stress imposed on the solder joints during thermal cycling and shock loads during impacts. The latter is of particular importance for hand held consumer products which are assembled using lead free solders. This is because most of the lead free solders currently being used offer a much lower ductility than the traditional near eutectic tin-lead alloys which, until recently, have been almost universally used in electronics assembly. However, these advantages may also be important for harsh environment applications where tin-lead solders are still currently being used, such as in aerospace, defence and automotive electronics applications, and may allow more widespread adoption of BGA/CSP for such safety critical applications. The increased compliance of a polymer cored ball may also eliminate the requirement for underfilling of components in some products, which will reduce assembly costs and facilitate rework/repair operations.

Modelling techniques offer a cost effective method to achieving a detailed understanding of the effect of particle properties on performance, and therefore offer a route to appropriate materials selection for the polymer spheres and their conductive coatings, and for establishing optimum values for design parameters such as ball diameter, conductive coating thickness, solder pad diameter, and solder volumes. Important inputs to such models include materials properties and the geometry, however the solder fillet geometry depends not only upon the geometric design parameters, but also on the properties of the solder and surfaces wetted by the solder. The Surface Evolver, a computational modelling tool capable of predicting the equilibrium shape of free liquid surfaces, is therefore used to predict the solder fillet shape for a polymer cored BGA assembly. These Evolver results are then used as an input to analytical and computational models of the thermal and electrical resistances of a typical BGA assembly manufactured using polymer cored balls. These models are compared with models of the same device assembled using conventional solder balls.

\section{Solder fillet geometry prediction}

For a conventional BGA assembly if the weight of the component is insufficient to have a significant effect on the solder joint height, then the solder fillet shape following reflow can be approximated as a truncated sphere. For a given volume of solder and diameter of the solder pads it is therefore straightforward to estimate the joint height and diameter. For the work reported in this paper the geometry typical of a commonly used $1.27 \mathrm{~mm}$ pitch, 593 I/O, BGA package has been used. The solder pad diameter is $0.7 \mathrm{~mm}$ and the assembled standoff height is $0.5 \mathrm{~mm}$ with a sphere diameter of $0.8 \mathrm{~mm}$ for a solder volume of $0.24 \mathrm{~mm}^{3}$. 
For the polymer cored balls the stand off height of the components is however determined by the ball diameter rather than the volume of the solder joints. The solder fillet shape will however still be important, particularly to the stress distribution within the assembly, but also to a significant extent to the electrical and thermal conductivity, for which the solder joint fillet height would be expected to be the most significant feature. Prediction of the geometry of the fillets is however more difficult using analytical approaches, as they are formed between the flat pad surface and the spherical surface of the conductor particle. The Surface Evolver [2] is a public domain computer program which may be used to predict the equilibrium shape of liquid surfaces where their geometry is principally determined by surface tension forces and has been used previously in predicting solder joint shapes. Evolver is therefore used here to estimate the solder fillet geometry for a similar BGA geometry. The conductor particle size for the polymer cored interconnects determines both the standoff height and the joint diameter and in choosing the particle diameter it must be decided whether to aim for the same sphere diameter as the solder ball BGA, the same standoff height, or some other figure. In this case it was decided to choose the same overall sphere diameter of $0.8 \mathrm{~mm}$ together with the same $0.7 \mathrm{~mm}$ diameter solder pads, as this would be expected to provide a worst case comparison with the solder ball BGA assembly. The solder volume in each solder fillet was chosen to be representative of that of a typical stencil printed solder deposit over the whole pad area, which for a $50 \%$ solder paste metal content and $200 \mu \mathrm{m}$ thick stencil is $0.0385 \mathrm{~mm}^{3}$. The wetting angles of typical lead free solders have been found to be somewhat higher than for tin-lead solder. For example Siewert et al. [3] reported the wetting angle of the SnAgCu eutectic to be 32-36 degrees. A wetting angle of 34 degrees was therefore chosen for the model.

Figure 1 shows the results of an Evolver model of the solder fillet shape for these parameters. The model predicts a solder joint fillet height (maximum distance from solder pad) of $0.171 \mathrm{~mm}$. This height is used as a baseline in the following thermal and electrical modelling of the interconnect.

\section{Thermal and electrical modelling}

To compare the electrical and thermal performance of the proposed polymer cored interconnects with the traditional solid solder balls only requires modelling of the interconnects themselves to generate a comparative thermal/electrical resistance for the two design approaches, as the remainder of the electronic packaging and interconnection structure will remain the same.

Steady state heat transfer and electrical current flow may both be modelled using similar approaches and a number of techniques for modelling of these types of problems exist. Analytical models may be used to model simple geometries with good accuracy, and can be used to rapidly assess the effects of design parameters and to generate design curves. However more complex geometries require more sophisticated modelling approaches, such as finite element analysis (FEA).

The individual BGA interconnects are rotationally symmetric about their vertical axis and, if the thermal or electrical loads can be assumed to be uniformly distributed over the solder pad areas, the interconnect may be modelled using axisymmetric approaches. If the solder pad sizes and, for the polymer cored interconnects, volumes of solder in the fillets above and below the ball can also be assumed to be equal then a horizontal plane of symmetry can also be used to further reduce the model complexity.

The following sections will describe the use of axisymmetric analytical and FEA approaches to:

(i) modelling of the thermal and electrical resistances of a typical conventional BGA geometry and their sensitivity to design parameters; modelling of the electrical behaviour of an equivalent polymer cored BGA; and modelling of the thermal behaviour of the polymer cored BGA.

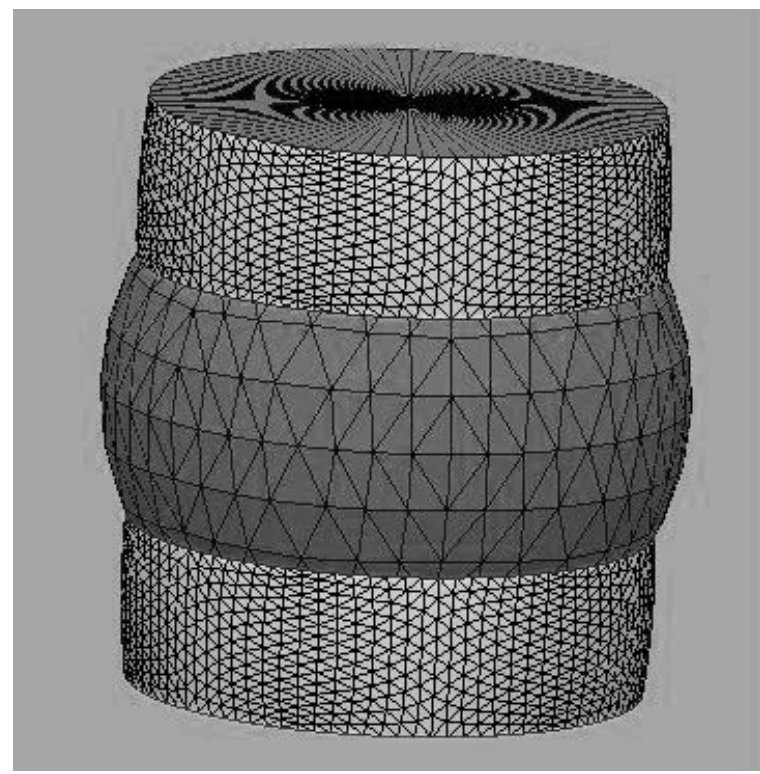

Figure 1. Evolver model of the polymer cored BGA interconnect

\section{Modelling of a traditional BGA interconnect}

The thermal or electrical resistance of a single homogenous solder BGA ball can both be estimated using the same modelling approaches. An upper limit can be quickly obtained by simply treating the interconnect as a cylindrical column of solder with a diameter equal to the solder pad diameters and length equal to the component standoff height i.e. the electrical $(\Omega)$ or thermal $\left({ }^{\circ} \mathrm{C} / \mathrm{W}\right)$ resistance, $R$, can be roughly approximated as:

$$
R=\frac{h}{K \cdot A}=\frac{h}{K \cdot \pi \cdot r_{p}{ }^{2}}
$$


where $h$ is the joint height, $K$ is the conductivity of the solder $(\mathrm{W} / \mathrm{mK}$ for thermal or $1 / \Omega \mathrm{m}$ for electrical i.e. the reciprocal of the more commonly used electrical resistivity with a unit of $\Omega \mathrm{m}), A$ is the cross sectional area of the joint and $r_{p}$ is the radius of the solder pad.

In practice the resistance will be lower than predicted by equation (1), due to the greater cross sectional area of the truncated solder sphere other than at the interface with the solder pads. A more detailed analytical model of conduction through these spheres can be derived, but it must still be assumed that both the temperature/voltage and heat flux/current are uniformly distributed over the pad area. Figure 2 shows the geometry of half of the solder ball used in the following derivation of the resistance.

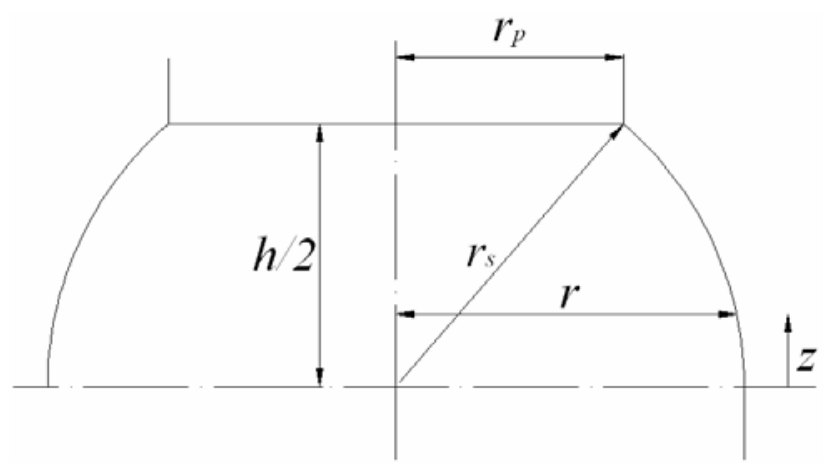

Figure 2. Geometry of the solid solder BGA ball

The radius, $r$, of any horizontal slice through the ball at height $z$ is given by:

$$
r=\sqrt{r_{s}^{2}-z^{2}}
$$

However:

$$
r_{s}=\sqrt{(h / 2)^{2}+r_{p}^{2}}
$$

So, combining (2) and (3):

$$
r=\sqrt{(h / 2)^{2}+r_{p}^{2}-z^{2}}
$$

And the cross sectional area, $A$, of any slice through the ball at a height $z$ is:

$$
A=\pi\left(r_{p}^{2}+\frac{h^{2}}{4}-z^{2}\right)
$$

The increment in the resistance, $\delta R$, for an increment in $z$ is therefore:

$$
\delta_{R}=\frac{1}{\pi \cdot K\left(r_{p}^{2}+\frac{h^{2}}{4}-z^{2}\right)} \delta z
$$

The total resistance of the ball, $R$, is twice that obtained by integrating this equation over half the height, i.e:

$$
\begin{aligned}
& R=\frac{2}{\pi \cdot K} \int_{z=0}^{z=h / 2} \frac{d z}{r_{p}^{2}+h^{2} / 4-z^{2}} \\
& R=\frac{2}{\pi \cdot K}\left[\frac{1}{2 \sqrt{r_{p}^{2}+h^{2} / 4}} \ln \left|\frac{z+\sqrt{r_{p}^{2}+h^{2} / 4}}{z-\sqrt{r_{p}^{2}+h^{2} / 4}}\right|\right]_{0}^{h / 2} \\
& R=\frac{2}{\pi \cdot K}\left[\frac{1}{2 \sqrt{r_{p}^{2}+h^{2} / 4}} \ln \left|\frac{h / 2+\sqrt{r_{p}^{2}+h^{2} / 4}}{h / 2-\sqrt{r_{p}^{2}+h^{2} / 4}}\right|\right]
\end{aligned}
$$

A third approach to modelling the resistance, which for this simple geometry would be expected to yield similar results to the analytical model presented above, is finite element analysis. Figure 3 shows a finite element model of the solder ball. For this model it must be decided whether to apply a fixed temperature/voltage differential across the solder ball and calculate the average flux/current through it, or apply a uniform flux/current to the solder pad and calculate the temperature/voltage differential. In this case a fixed temperature/voltage was applied and the resulting heat flux/current was obtained from the model and then Ohm's Law used to calculate the resistance.

Table 1 compares the electrical and thermal resistances calculated using these three approaches for a thermal conductivity of $50 \mathrm{~W} / \mathrm{mK}$ and electrical resistivity of $15 \times 10^{-8} \Omega \mathrm{m}$. The results confirm that the detailed analytical model provides results comparable to the FEA model, but that the cylindrical model over estimates the resistance by around $20 \%$. The detailed analytical model has therefore been used to evaluate how the pad size and standoff height affect the thermal resistance, as presented in figure 4. The electrical resistance will vary in proportion to the thermal resistance and so is not separately calculated. It can be seen that, for a given pad size, the resistance initially increases with joint height, but it then reaches a maximum for a height around 1.5 times the pad diameter before then falling for further increases in height. This behaviour can be attributed to the joint cross sectional area initially being constrained by the pad diameter, but as the height increases the area begins to grow more quickly and for larger heights the area will grow with the square of the height, thereby reducing the resistance. In real applications the typical joint height to pad ratios will be well below that for the maximum resistance and the joint height should therefore be minimised where possible. 


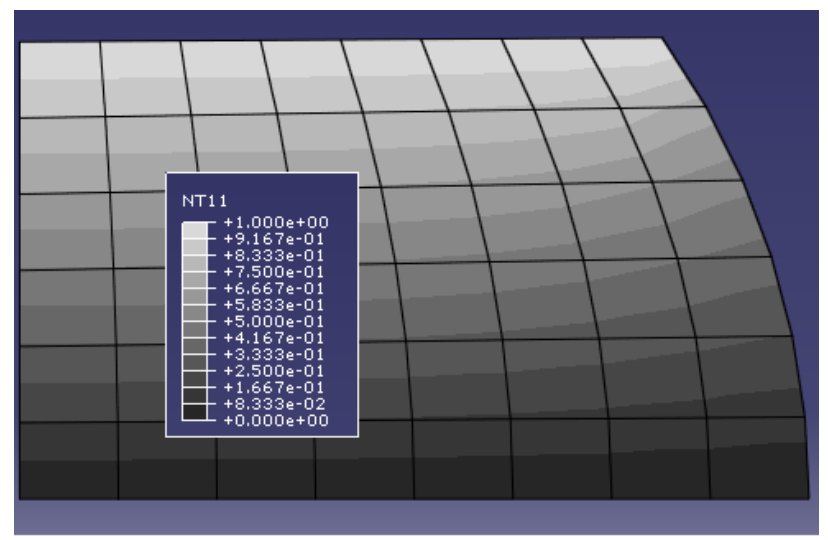

Figure 3. FEA model of the solder ball

\begin{tabular}{|c|c|c|c|}
\hline & $\begin{array}{c}\text { Cylinder } \\
\text { model }\end{array}$ & $\begin{array}{c}\text { Detailed } \\
\text { analytical } \\
\text { model }\end{array}$ & $\begin{array}{c}\text { FEA } \\
\text { model }\end{array}$ \\
\hline $\begin{array}{c}\text { Thermal } \\
\text { resistance } \\
\left({ }^{\circ} \mathrm{C} / \mathrm{W}\right)\end{array}$ & 26.0 & 19.7 & 20.1 \\
\hline $\begin{array}{c}\text { Electrical } \\
\text { resistance } \\
(\mathrm{m} \Omega)\end{array}$ & 0.195 & 0.147 & 0.151 \\
\hline
\end{tabular}

Table 1. Comparison of modelling results for a single BGA solder ball

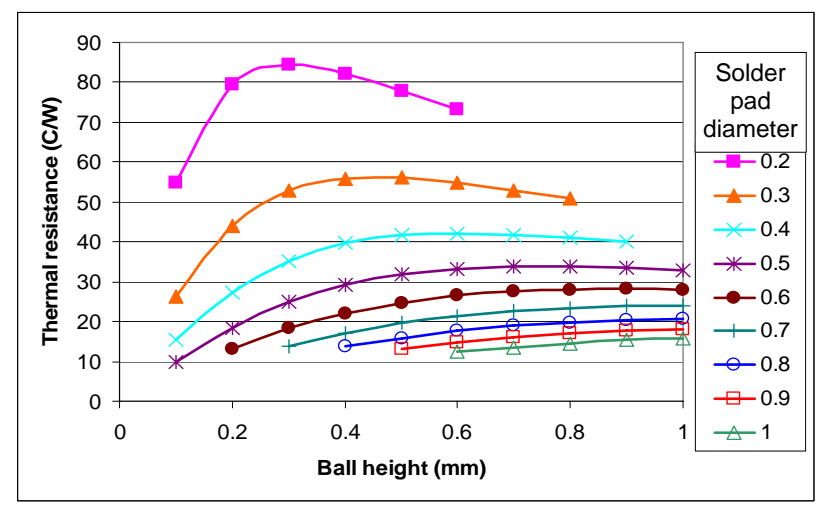

Figure 4. Effect of solder pad diameter and joint height on predicted solder ball thermal resistance

\section{Electrical modelling of the polymer cored BGA interconnect}

Figure 5 shows the geometry of the polymer cored BGA and its solder joint. In developing a simple analytical model for the electrical resistance this geometry can conveniently be split into three resistive components in series:

$R_{1}$, the resistance of the part of the conductive coating of the ball which is not wetted by solder;

$R_{2}$, which is the parallel resistance of the part of the solder fillet and part of the conductive coating from $h_{s}$ to $\mathrm{r}_{\mathrm{p}}$; and
$R_{3}$, the small additional resistance of the remaining part of the solder fillet and conductive coating in parallel from $r_{p}$ to $r_{b}$.

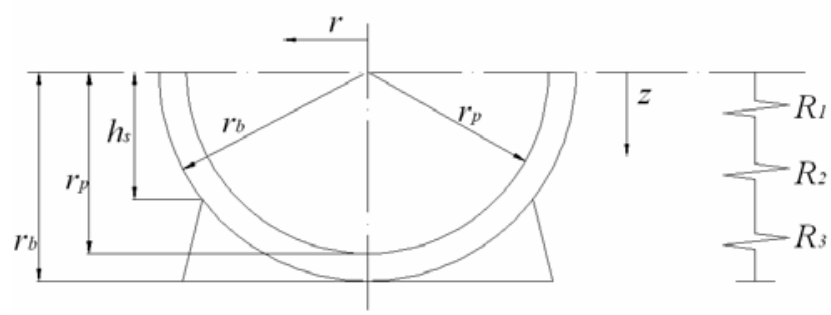

\section{Figure 5. Geometry of the polymer cored BGA} interconnect

Estimation of $\boldsymbol{R}_{\mathbf{1}}$. In this region the current will travel parallel to the surface of the sphere and as the thickness of the metal coating can be assumed to be small relative to the ball radius the area of conduction, $A$, at any radius, r, will be:

$$
A=2 \cdot \pi \cdot t \cdot r
$$

where $t$ is the metallic coating thickness, i.e:

$$
t=r_{b}-r_{p}
$$

and:

$$
r=\sqrt{r_{b}^{2}-z^{2}}
$$

The increment in the resistance, $\delta R$, for an increment in $z$ is therefore:

$$
\delta R=\frac{1}{2 \cdot \pi \cdot K \cdot t \cdot \sqrt{r_{b}^{2}-z^{2}}} \delta Z
$$

and the resistance, $R_{1}$, is therefore obtained by integration:

$$
\begin{aligned}
& R_{1}=\frac{1}{2 \cdot \pi \cdot K \cdot t} \int_{z=0}^{z=h_{s}} \frac{1}{\sqrt{r_{b}^{2}-z^{2}}} d z \\
& R_{1}=\frac{1}{2 \cdot \pi \cdot K \cdot t}\left[\sin ^{-1}\left(\frac{z}{r_{b}}\right)\right]_{0}^{h_{s}} \\
& R_{1}=\frac{1}{2 \cdot \pi \cdot K \cdot t}\left[\sin ^{-1}\left(\frac{h_{s}}{r_{b}}\right)\right]
\end{aligned}
$$

Estimation of $\boldsymbol{R}_{\boldsymbol{2}}$. Deriving an appropriate analytical expression for $R_{2}$ is considerably more difficult as it is composed of two materials and the geometry of the solder fillet is more complex. Incorporating an accurate representation of the solder geometry would result in an equation requiring numerical integration. Some significant simplification of this geometry however yields a more manageable model. Firstly, the area of the metal 
coating on the polymer particle is calculated, then the area of the solder is roughly calculated and finally these are combined in an integral to calculate the resistance. The total area, $A_{T}$, of any slice through the ball at a height $\mathrm{z}$ is:

$$
A_{T}=\pi\left(r_{b}^{2}-z^{2}\right)
$$
$z$ is:

And the area, $A_{p}$, of the slice of polymer core at height

$$
A_{P}=\pi\left(r_{p}^{2}-z^{2}\right)
$$

The area of any slice of the metal coating, $A_{m}$ between the centre line and $r_{p}$ is:

$$
A_{M}=A_{T}-A_{p}
$$

And by combining equations 13, 14 and 15 it can be seen that the area of the slice is independent of $\mathrm{z}$ :

$$
A_{M}=\pi\left(r_{b}^{2}-r_{p}^{2}\right)
$$

As mentioned earlier the area of the solder, $A_{S}$, must be approximated to yield an exactly integrable result. For the geometry of the particular BGA being studied here the radius of the contact between the solder and ball, $r_{s}$, is $0.328 \mathrm{~mm}$, which is similar to that of the solder pad $(0.35 \mathrm{~mm})$ so the shape of the solder surface can be roughly represented as a vertical line at $r_{s}$. By using this approximation the area of any slice through the solder is:

$$
A_{s}=\pi r_{s}^{2}-\pi\left(r_{b}^{2}-z^{2}\right)
$$

where:

$$
r_{s}=\sqrt{r_{b}^{2}-h_{s}^{2}}
$$

so:

$$
\begin{aligned}
& A_{S}=\pi\left(\left(r_{b}^{2}-h_{s}^{2}\right)-\left(r_{b}^{2}-z^{2}\right)\right) \\
& A_{S}=\pi\left(z^{2}-h_{s}^{2}\right)
\end{aligned}
$$

The increment in the resistance, $\delta R_{2}$, for an increment in $z$ is:

$$
\delta R_{2}=\frac{1}{K_{M} \cdot A_{M}+K_{S} \cdot A_{S}} \delta z
$$

where $K_{M}$ and $K_{S}$ are the electrical conductivities of the metal coating and solder respectively. Combining equations 16, 19 and 20 and integrating gives:

$$
\begin{aligned}
& R_{2}=\frac{1}{\pi} \int_{z=h_{s}}^{z=r_{p}} \frac{1}{K_{M}\left(r_{b}^{2}-r_{p}^{2}\right)+K_{S}\left(z^{2}-h_{s}^{2}\right)^{d z}} \\
& R_{2}=\frac{1}{K_{S} \cdot \pi} \int_{z=h_{s}}^{z=r_{p}} \frac{1}{b^{2}+z^{2}} d z
\end{aligned}
$$

where:

$$
b^{2}=K_{M}\left(r_{b}^{2}-r_{p}^{2}\right) / K_{S}-h_{s}^{2}
$$

so finally:

$$
R_{2}=\frac{1}{K_{S} \cdot \pi \cdot b}\left[\tan ^{-1}\left(\frac{z}{b}\right)\right]_{h_{s}}^{r_{p}}
$$

The metal coating on the polymer spheres is assumed to be copper with a thickness of $10 \mu \mathrm{m}$ and an electrical resistivity of $1.58 \times 10^{-8} \Omega \mathrm{m}$, and the solder is again assumed to have a resistivity of $15 \times 10^{-8} \Omega \mathrm{m}$ [3]. Calculation of $\mathrm{R}_{1}$ for these parameters yields a figure of $0.153 \mathrm{~m} \Omega$, whilst the calculated value for $\mathrm{R}_{2}$ is $0.068 \mathrm{~m} \Omega$. $\mathrm{R}_{3}$ is ignored as it will only be a few micro-ohms. The total resistance predicted by this analytical model is therefore two times the sum of $R_{1}$ and $R_{2}$ or $0.442 \mathrm{~m} \Omega$.

An FEA model of this situation has also been performed. In order to avoid the difficulty in meshing the area where the conductor particle contacts the solder pad a small gap of $10 \mu \mathrm{m}$ has been introduced between them. The solder surface tension will try to pull all of the conductor particles into contact with their solder pads, however any non-planarity of the substrate or component may in practice result in such small gaps occurring for a significant proportion of the balls. The additional series thermal resistance of this thin solder layer can be estimated to be only about $4 \mu \Omega$. The shape of the solder fillet is also approximated as a straight line rather than using the curvature predicted by Evolver. This simplified model construction and would not be expected to make a substantial difference to the resistance results, but future mechanical analysis will require more detailed consideration of this geometry. Figure 6 shows the FEA model, for which the predicted resistance was $0.54 \mathrm{~m} \Omega$. This is $22 \%$ higher than the analytical model prediction and this difference indicates the much greater difficulty in developing an accurate analytical model for this geometry compared with the solid solder balls., However, despite the poorer accuracy of the analytical model, it may be cautiously applied in the evaluation of the effects of design variables on the resistance. Figure 7 shows the results of using the analytical model to evaluate the effects of the metal coating thickness on resistance. Parametric FEA modelling is a more robust option for such evaluations, but are much more time consuming.

\section{Thermal modelling of the polymer cored BGA interconnect}

The electrical resistivity of the polymer sphere material will be many orders of magnitude greater than that of its metallic coating or of the solder joint material i.e. $10^{12}-10^{14} \Omega \mathrm{m}$ is typical for polymers compared with the $10^{-7}-10^{-9} \Omega \mathrm{m}$ typical of metals. The presence of the polymer was therefore ignored in the electrical resistance calculations. However the thermal conductivities of 
typical polymer materials are only a few orders of magnitude less than those of metals and it is therefore important to consider whether the polymer sphere may have a significant effect on the thermal resistance of the interconnect.

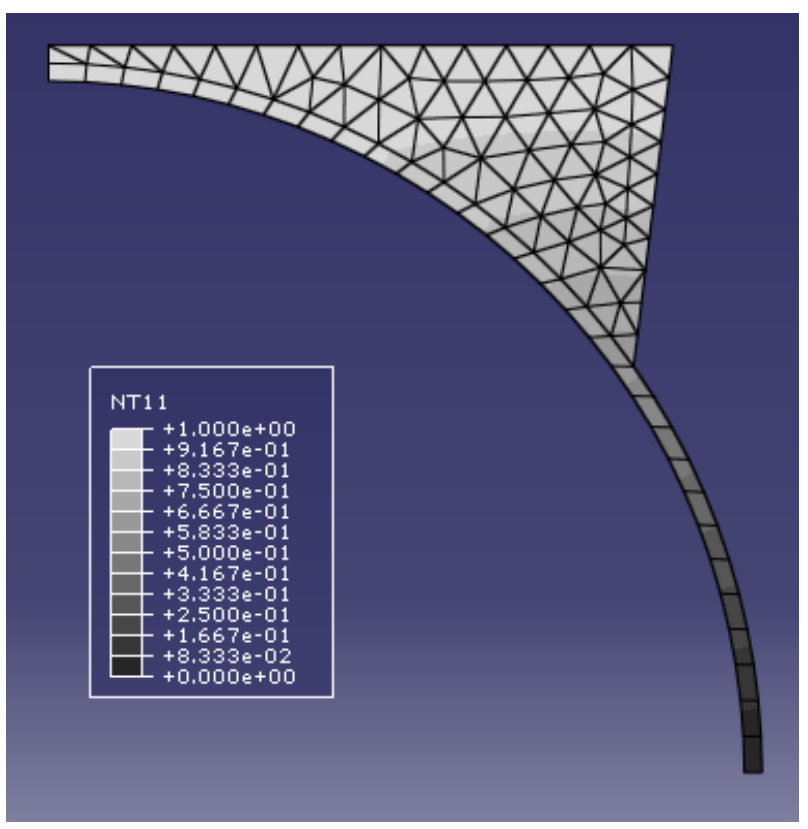

Figure 6. FEA model of electrical conduction in the polymer cored BGA interconnect

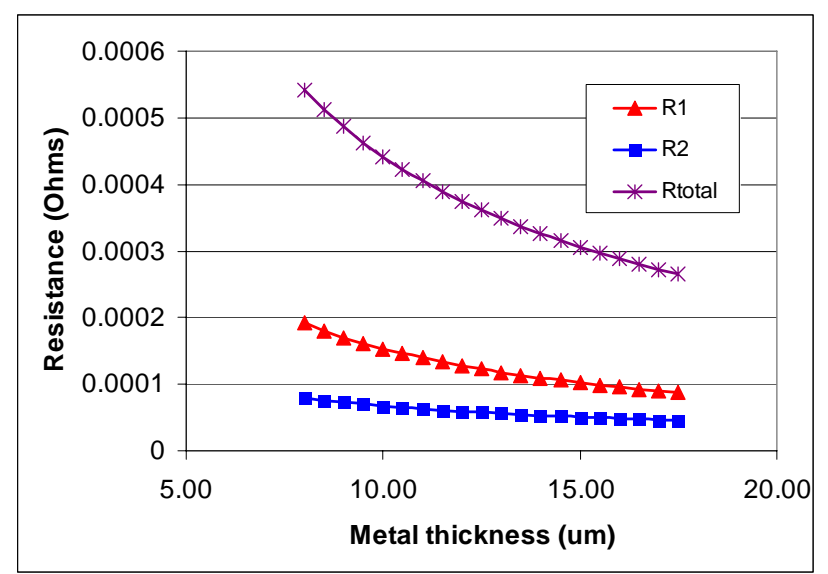

Figure 7. Effect of metal coating thickness on the electrical resistance of a polymer cored BGA interconnect

The thermal resistance of the polymer cored BGA has only been modelled using FEA due to the even greater difficulty in accurately representing the geometry with an analytical model. Figure 8 shows an example FEA model using a thermal conductivity for the copper coating of the polymer particle of $385 \mathrm{~W} / \mathrm{mK}$ and for the polymer of $0.2 \mathrm{~W} / \mathrm{mK}$, a figure typical of unfilled polymers. The same geometrical approximations were applied as for the electrical resistance modelling. The predicted thermal resistance in this case was $84.8^{\circ} \mathrm{C} / \mathrm{W}$. Increasing the thermal conductivity of the polymer by an order of magnitude to $2.0 \mathrm{~W} / \mathrm{mK}$ reduced the thermal resistance to $79.2^{\circ} \mathrm{C} / \mathrm{W}$, whilst reducing it to $0 \mathrm{~W} / \mathrm{mK}$ only increased it to $85.2^{\circ} \mathrm{C} / \mathrm{W}$. This shows that while the thermal conductivity of the polymer does have a small effect on the overall thermal resistance of the interconnect it can probably be neglected in any further design studies.

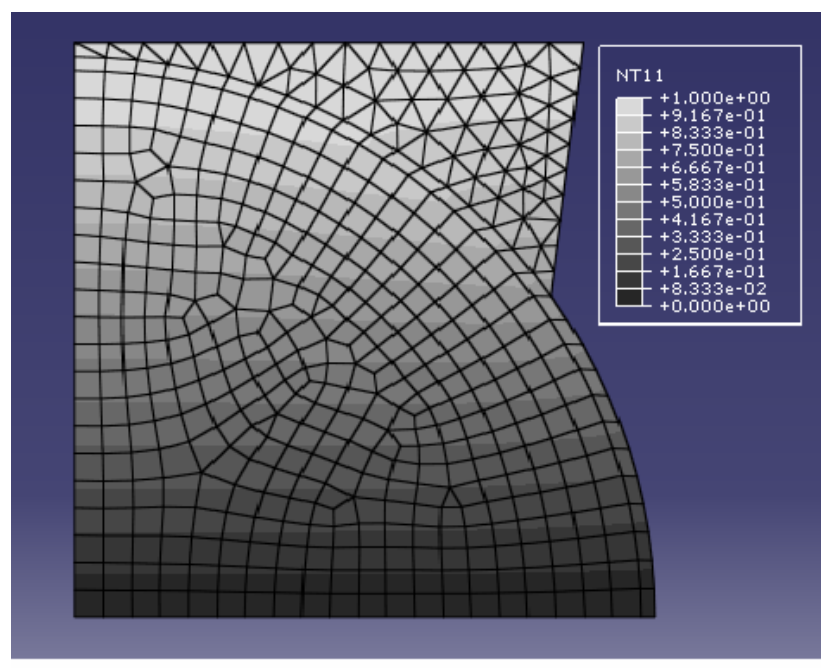

Figure 8. FEA model of temperature distribution in the polymer cored BGA interconnect

\section{Discussion}

The results of this work have shown that the use of polymer cored BGA balls will result in some increase in package electrical and thermal resistances. However, the predicted increases in electrical trace resistances of around $0.4 \mathrm{~m} \Omega$ are unlikely to present an issue, as this increment in resistance is very small in comparison with typical substrate trace resistances. For example the trace resistance for a $1 \mathrm{oz}(35 \mu \mathrm{m})$ thick copper track $1 \mathrm{~mm}$ wide is $0.45 \mathrm{~m} \Omega / \mathrm{mm}$. The predicted increase in thermal resistance is also not likely to present a significant issue. For example, if it assumed that all of the heat generated in the package flows into the PCB by conduction through the 593 identical interconnects then a rough estimate of the increase in overall package thermal resistance, $\Delta R$, can be made by dividing the increase in the individual interconnect resistance by the total number of interconnects, i.e. $\Delta R=(84.8-20.1) / 593=0.11^{\circ} \mathrm{C} / \mathrm{W}$. This is only a small fraction of typical total thermal resistances from die to ambient and will only generate a significant additional temperature rise for components generating substantial powers. However such components are likely to have additional thermal management systems in place, and the proportion of the heat flowing through the interconnects into the substrate will be less important to overall thermal performance.

Another interesting observation that can be made from the analytical modelling results is the effect on resistance of scaling down the size of the BGA ball, whilst keeping the same proportions between ball and solder fillet. For such scaling the resistance of a solder ball will increase 
with the reciprocal of the ball size, as the cross sectional area scales with $r^{2}$ and conduction length with $r$. For the polymer core ball $R_{2}$ will also scale with the reciprocal of ball radius in the same way. However $R_{1}$ will remain constant, which is the larger part of the total resistance as long as metal thickness is small compared to the ball radius. Scaling down the ball size will therefore proportionately reduce the extra contact resistance for the polymer core ball as compared to the solid solder ball.

\section{Conclusions}

The results of this work show that although replacing solid solder balls in BGA applications with polymer cored balls will result in significant increases in the thermal and electrical resistance of the individual interconnects, that the effect of these increases on overall performance will be small enough not to act as a deterrent to adoption of polymer cored BGA balls. Future work will focus on evaluating the mechanical performance of the interconnections in order to identify how the materials and design of polymer cored interconnects can be optimised to maximise the reliability benefits of this technology.

\section{Acknowledgments}

The authors acknowledge the financial support of the Research Council of Norway, through their Nanotechnology and New Materials program.

\section{References}

1. Movva, S. and Aguirre, G. "High reliability second level interconnects using polymer core BGAs" Proceedings of the 54th Electronic Components and Technology Conference, Las Vegas, USA, June 2004, Vol. 2, pp 1443 - 1448

2. Brakke, K. “The Surface Evolver”, Experimental Mathematics, Vol. 1, No. 2 (1992), pp 141-165

3. Siewert, T., Liu, S., Smith, D.R. and Madeni, J.C. "Database for Solder Properties with Emphasis on New Lead-free Solders" National Institute of Standards and Technology, Colorado, 2003, Release 4.0 
